\title{
Research Square \\ Retrospective analysis of primary neuroendocrine tumors of the ovary: Management and outcomes
}

li pang

Shengjing Hospital of China Medical University

zhiqiang guo ( $\sim$ r4849330330@163.com )

Shengjing Hospital of China Medical University

\section{Research}

Keywords: ovarian neuroendocrine tumor, chemotherapy, SEER database, ovarian cancer

Posted Date: August 18th, 2021

DOI: https://doi.org/10.21203/rs.3.rs-814541/v1

License: (c) (i) This work is licensed under a Creative Commons Attribution 4.0 International License. Read Full License 


\section{Abstract \\ Background}

Due to the low incidence of ovarian neuroendocrine tumors (NETs), clinicians may be unaware of appropriate treatments for the disease and factors influencing patient prognosis, which may cause them to miss the window of opportunity for treatment. Moreover, there is currently no recognized first-line treatment strategy, and no studies have reported prognostic statistics derived from large samples. This retrospective study aimed to investigate the clinical behavior of ovarian NETs.

\section{Methods}

The Surveillance, Epidemiology, and End Results database was used to identify women diagnosed with ovarian NETs from 2004 to 2015. Overall survival (OS), cancer-specific survival (CSS), and independent prognostic factors for ovarian NETs were evaluated. The effects of different treatments on prognosis were also compared, as were OS and CSS rates for histological subtypes.

\section{Results}

The 5-year OS rates were $83.3 \%, 30.0 \%, 20.3 \%$, and $9.8 \%$ for patients in stages I $(\mathrm{n}=159)$, II $(\mathrm{n}=23)$, III ( $\mathrm{n}$ $=101)$, and IV $(n=148)$, respectively. The 5-year CSS rates were $85.6 \%, 41.7 \%, 21.2 \%$, and $9.8 \%$ for patients in stages I-IV, respectively. Age, American Joint Committee on Cancer (AJCC) stage, lymph node metastasis, treatment, and histological type were related to poor OS and CSS. In the early stage, the 5year OS and CSS rates were $97.03 \%$ and $96.90 \%$, respectively. For patients in the advanced stage receiving comprehensive treatment (surgery + chemotherapy + radiotherapy), 5-year OS and CSS rates were $72.9 \%$ and $70.00 \%$, respectively. When comparing low- and high-grade neuroendocrine carcinoma, the 5 -year OS rates were $93.96 \%$ vs. $7.01 \%$, 5-year CSS rates were $97.44 \%$ vs. $7.31 \%, 10$-year OS rates were $93.56 \%$ vs. $2.34 \%$, and 10 -year CSS rates were $97.44 \%$ vs. $4.88 \%$, respectively.

\section{Conclusion}

Age, AJCC stage, treatment, and histological type are independent prognostic factors of ovarian NETs. Prognosis is relatively good for early-stage cases treated with surgery alone, whereas more comprehensive treatment is required to improve prognosis for advanced cases. Future studies should focus on the development of individualized treatment strategies for prolonging survival time in patients with ovarian NETs.

\section{Background}


Derived from neuroendocrine cells, neuroendocrine tumors (NETs) are aggressive diseases that often occur in the gastrointestinal tract, pancreas, and lungs. Cases occurring in other tissues and organs are rare, especially in the female reproductive tract [1]. Ovarian NETs account for only $1-2 \%$ of malignant ovarian tumors [2,3]. At present, these tumors can be roughly divided into carcinoid, atypical carcinoid (ACT), small-cell carcinoma (SCCO), and large-cell neuroendocrine carcinoma (LCNEC) types [3]. According to World Health Organization (WHO) regulations, non-small-cell neuroendocrine carcinoma (NSCNEC) is synonymous with LCNEC [4], and the first two types are classified as low-grade neuroendocrine tumors (LGNET), whereas the latter two are classified as high-grade neuroendocrine carcinoma (HGNEC) [4]. HGNEC is considered more aggressive than LGNET [4-6].

Due to the low incidence of ovarian NETs, clinicians may be unaware of appropriate treatments for the disease and factors influencing patient prognosis, which may cause them to miss the window of opportunity for treatment. Furthermore, few cases of ovarian NETs have been reported in the literature, and such cases are often reported in small series only. Moreover, there is currently no recognized first-line treatment strategy, and no studies have reported prognostic statistics derived from large samples.

The Surveillance, Epidemiology, and End Results (SEER) database is considered a powerful tool for identifying population characteristics and studying the long-term prognosis of rare tumors [7]. In the present study, we used the SEER database to extract relevant patient information and analyze the clinical behavior and prognostic factors of ovarian NETs. In addition, we systematically assessed the prognosis of ovarian NET and evaluated the effect of ovarian NET treatment on prognosis. Finally, we compared our findings between LGNET and HGNEC.

\section{Materials And Methods}

\subsection{Eligibility criteria and data collection}

This retrospective observational cohort study utilized the SEER database of the National Cancer Institute [7]. The SEER program is the largest population-based tumor registration system in the United States. The SEER database was launched in 1973 and has a history of more than 40 years of operation, covering approximately $34.6 \%$ of the U.S. population in the most recent update. It is considered a powerful tool for identifying population characteristics and studying the long-term prognosis of rare tumors. Patients were identified using the SEER database, and those histologically diagnosed with ovarian NETs from 2004 to 2015 who met the following criteria were considered eligible: primary malignant tumor in the ovary (ICD0-3/WHO 2008 site code), carcinoid tumor (8240/3), atypical carcinoid tumor (8249/3), large-cell carcinoma (8012/3), large-cell neuroendocrine carcinoma (8013/3), non-small-cell carcinoma (8046/3), and small-cell carcinoma (8041/3). The exclusion criteria were as follows: diagnosis of carcinoma in situ and cases in which the ovarian NET was not the first tumor.

SEER ${ }^{\star S t a t ~}$ 8.3.8 software (https://seer.cancer.gov/data/) was used to generate case listings. The deidentified data in the SEER database are publicly available, and thus their use is exempt from review by 
the Shengjing Hospital of China Medical University Institutional Review Board. The same software was used to record patient information, including demographic characteristics, clinical pathology findings, and treatment parameters. Staging information was determined based on the American Joint Committee on Cancer (AJCC) staging system.

\subsection{Clinical and demographic characteristics}

Demographic data in this analysis included age at diagnosis ( $\leq 49,50-69$, and $\geq 70$ years), AJCC stage (I, IA, IB, IC, II, IIA, IIB, IIC, III, IIIA, IIIB, IIIC, IV), year of diagnosis (2004-2009, 2010-2015), grade (welldifferentiated, moderately differentiated, poorly differentiated, undifferentiated, differentiated), tumor size ( $\leq 5.0 \mathrm{~cm},>5.0 \mathrm{~cm}$, unknown), nodal metastasis (negative, positive, not examined, and unknown), sampled pelvic nodes $(1-9,10-19, \geq 20$, not examined, unknown), distant metastasis (bone, brain, liver, lung, none, unknown), treatment (surgery alone, surgery + chemotherapy [CTX], surgery + concurrent chemoradiotherapy [CCRT], CTX alone, radiotherapy [RT] + CTX, RT alone, no treatment). Follow-up time after diagnosis, life status, and cause of death were collected from the database to evaluate survival due to disease (i.e., cancer-specific survival [CSS]) and overall survival (OS).

\subsection{Statistical analysis}

Categorical data are presented as numbers and percentages $(\mathrm{N}, \%)$. The chi-square test or Fisher's exact test was used to compare the clinical and demographic characteristics of women with LGNET and HGNEC. Univariate and multivariate Cox regression analyses were used to determine independent predictors of OS and CSS in patients with ovarian NETs. OS and CSS were calculated using KaplanMeier curves, and the log-rank test was used for comparison. All data were analyzed using SPSS software (version 23.0; SPSS, Chicago, IL, USA). GraphPad Prism (8.3.0 GraphPad Software, San Diego, California, USA) was used to draw Kaplan-Meier survival curves, and $\mathrm{P}$ values $<0.05$ were considered statistically significant.

\section{Results}

\subsection{Patients}

A total of 431 cases of ovarian NETs in the SEER registry met the inclusion criteria. Table 1 summarizes the characteristics of patients with ovarian NETs, including age, year at diagnosis, AJCC stage, tumor size, grade, nodal metastasis, sampled pelvic nodes, distant metastasis, and treatment. There were 124 cases of LGNET (carcinoid: 118, ACT: 5) and 254 cases of HGNEC (SCNEC: 124, LCNEC: 130, NET-not classified: 54$)$.

The treatment for each stage is summarized in Additional File 1. Among the 431 included patients, 162 underwent surgery alone. Among the 159 patients in stage I, surgery alone was the primary treatment in 125 patients (78.6\%). Most of the 116 patients who underwent surgery + CTX (69.8\%) were in the advanced stage, with 46 in stage III and 35 in stage IV. Seventeen patients underwent surgery + CCRT, whereas 62 patients received CTX only, and most of these patients $(91.9 \%)$ were also in the advanced 
stage (stage III: 18, stage IV: 39). Only eight patients in stage IV received RT + CTX. Four patients received RT only, including two patients in stage III and two patients in stage IV (Additional File 1).

\subsection{Survival curves}

Figure 1 shows the OS and CSS curves for patients in each stage. The 5-year OS rates for patients with stage I, II, III, and IV disease were $83.3 \%, 30.0 \%, 20.3 \%$, and 9.8\%, respectively. Relative to stage I, hazard ratios (HR) for death at stages II, III, and IV were 6.389 (95\% confidence interval [CI]: 1.631-25.03), 9.32 (95\% Cl: 5.745-15.130), and 12.12 (95\% Cl: 8.252-17.79). The 5-year CCS rates for patients with stages I, II, III, and IV were 85.6\%, 41.7\%, 21.2\%, and 9.8\%, respectively. Relative to stage I, HRs for death at stages II, III, and IV were 5.794 (95\% confidence interval [CI]: 1.320-25.44), 10.10 (95\% Cl: 6.123-16.67), and 13.45 (95\% Cl: 9.095-19.88). The figure in Additional File 2 shows the survival curves for patients in different stages. Although few patients were in stages IB and IIB, the figure shows that survival time still gradually decreased as stage increased.

Figure 2 shows the OS and CSS curves of patients with LGNET and HGNEC. The 5-year OS rates for LGNET and HGNEC were $93.96 \%$ and $7.01 \%$, respectively. Relative to LGNET, the HR for death due to HGNEC was 69.26 (95\% confidence interval [Cl]: 48.82-98.26). The 5-year CSS rates for LGNET and HGNEC were $97.43 \%$ and $7.31 \%$, respectively. In addition, relative to LGNET, the HR for death due to HGNEC was 135.7 (95\% confidence interval [CI]: 94.82-194.3) $(P<0.001)$.

\subsection{Prognostic factors}

To determine factors influencing prognosis in patients with ovarian NET, we selected histological type (LGNET vs. HGNEC), age, AJCC stage, tumor size, nodal metastasis, distant metastasis, and treatment as variables for univariate and multivariate analyses (Table 2). Multivariate analysis revealed that age, AJCC stage, nodal metastasis, treatment, and histological type were associated with poor OS and CSS. Tumor size and distant metastasis were not identified as prognostic factors.

\subsection{Treatment and prognosis}

The main surgical treatments included unilateral appendectomy, total double appendectomy, total hysterectomy + double appendectomy + pelvic lymph node dissection, ovarian cancer cytoreductive surgery, and adjuvant treatments, such as chemotherapy and radiotherapy.

In the early stage, 132 patients received surgical treatment only. Their 5-year OS and CCS rates were 97.03\% and $96.90 \%$, respectively. Thirty-five patients underwent surgery and CCRT. Five-year OS and CCS rates in these patients were $65.5 \%$ and $57.1 \%$, respectively. The survival rates of these two treatment regimens were better than those of the other treatment regimens. Ten patients in the advanced stage received surgery $+\mathrm{CTX}+\mathrm{RT}$, and 5-year OS and CCS rates were $72.9 \%$ and $70.0 \%$ in patients with advanced disease receiving comprehensive treatment. However, the 5-year OS and CCS rates were only $17.5 \%$ and $19.3 \%$ after surgery alone. Among patients with early-stage disease, 159 were in stage I, whereas 23 were in stage II. These findings indicate that prognosis was relatively good among patients 
with early-stage disease treated with surgery alone, whereas more comprehensive treatment was required (surgery + CTX + RT) to improve prognosis in patients with advanced-stage disease (Fig. 3).

\subsection{Comparison between histological subtypes}

LGNET ( $n=123)$ and HGNEC ( $n=254)$ were compared in terms of patient age, AJCC stage, grade, tumor size, nodal metastasis, sampled pelvic nodes, distant metastasis, treatment, and survival rate (Table 4). LGNET progressed slowly, and most patients with LGNET were in stage 1 (92.7\% LGNET vs. $14.2 \%$ HGNEC), whereas most with HGNEC were in stage III or IV (5.7\% LGNET vs. 79.9\% HGNEC). In addition, well- or moderately differentiated grades were observed more frequently in patients with LGNET (34.1\%) than in those with HGNEC $(0.0 \%, \mathrm{P}<0.001)$. Tumor size $\leq 5.0 \mathrm{~cm}$ was also more common in patients with LGNET (42.3\%) than in those with HGNEC (7.1\%). In addition, LGNET was associated with lower rates of distant (2.4\% LGNET vs. 9.4\% HGNEC, $\mathrm{P}<0.001)$ and nodal metastasis (0.8\% LGNET vs. $14.6 \%$ HGNEC, $\mathrm{P}$ $<0.001)$ than HGNEC. Most patients with LGNET were treated with surgery alone (94.35\%), whereas the most common treatment among patients with HGNEC was surgery + CTX (37.8\%). The 5- and 10-year OS and CSS rates were much higher for LGNET than for HGNEC (5-year OS: 93.56\% LGNET vs. 7.01\% HGNEC; 5-year CSS: $97.44 \%$ LGNET vs. 7.31\% HGNEC; 10-year OS: 93.56\% LGNET vs. 2.34\% HGNEC; 10-year CSS: $97.44 \%$ LGNET vs. $4.88 \%$ HGNEC).

\section{Discussion}

In this study, we investigated treatment strategies, prognostic factors, and outcomes in the largest cohort of patients with ovarian NETs to date. The key finding of the current study is that our data reveal that age, AJCC stage, treatment method, and histological type were identified as independent prognostic factors for ovarian NET. In addition, our analysis revealed that prognosis was relatively good among patients with early-stage disease treated with surgery alone, whereas more comprehensive treatment (surgery + $\mathrm{CTX}+\mathrm{RT}$ ) was required to improve prognosis in patients with advanced-stage disease. Prognosis was also poor for patients with HGNEC than for those with LGNET.

Given the rarity of ovarian NETs, the literature consists mostly of case reports and small case series [811]. Moreover, this disease entity encompasses several tumor types, with the largest single series reporting 58 cases of ovarian large-cell carcinoma [12]. The median survival time for patients with largecell neuroendocrine carcinoma of the ovary is 10 months. In their analysis of 329 cases, Soga et al. [13] divided patients into two carcinoid groups based on the presence or absence of a related dermoid. The authors reported 5-year survival rates of $84-93.7 \%$ for these two groups. In addition, there are 11 case reports [14], all of which discuss patients who underwent primary surgery. Five of these patients received adjuvant CTX with platinum therapy. The median overall survival time among 11 patients was 20 months. However, no previous large-sample studies have investigated ovarian NETs, highlighting the practical clinical significance of the current study. 
Establishing a diagnosis of ovarian NET can be challenging. Immunohistochemistry can help identify these tumors because they express markers of neuroendocrine differentiation, such as neuron-specific enolase (NSE), synaptophysin, chromogranin CD56, vimentin, and epithelial membrane antigen (EMA) [4, 15]. The diagnosis can be confirmed by immunohistochemistry using one or more standard neuroendocrine markers. At present, SMARCA4 [16-18] is recognized as a biological marker of hypercalcemic-type small-cell carcinoma. Unfortunately, there are currently no candidate drugs for targeted therapy in these patients.

Our multivariate analysis revealed that treatment options were related to prognosis. Patients with earlystage ovarian NETs experienced good survival outcomes after surgery alone. The 5-year OS and CSS rates were as high as $97.03 \%$ and $96.90 \%$, respectively. For patients with advanced-stage disease, comprehensive treatment (surgery $+\mathrm{CTX}+\mathrm{RT}$ ) was associated with improved survival rates, regardless of the type of ovarian NET. Ovarian carcinoids were also often small and unilateral tumors confined to the ovaries. Unilateral salpingo-oophorectomy also appears to be associated with high cure rates [19], although other gastrointestinal metastases must be excluded. For patients with metastatic disease [20], it is recommended to conduct a thorough evaluation using magnetic resonance imaging (MRI) or 111pentetide indium scanning to rule out the presence of other major sources (such as the gastrointestinal tract) [21], and to ensure prompt initiation of appropriate treatment. Some scholars [1] believe that somatostatin analogs (such as lanreotide and octreotide) should be considered if carcinoids are diagnosed, and that somatostatin analogs should be used before and during tumor resection to prevent carcinoid crisis complications [22]. In a study by Nasioudis et al. [19], the authors were unable to verify that CTX can prolong survival time in patients with stage II-IV carcinoids, although another study noted that the Ki-67 proliferation index may aid in the selection of patients likely to benefit from CTX [23]. However, reports on ACT are rare. Indeed, there were only five cases in our study. Because ACT is an extremely rare disease, little is known regarding its biological behavior [24]. Our results indicated that treatments were carried out in accordance with the carcinoid regimen, and that large-cell carcinoma and small-cell carcinoma of the ovary were uncommon and classified as HGNEC. The literature reports that prognosis is poor and that progression is rapid among these patients. Even when discovered early, the probability of metastasis or recurrence remains high [12, 15, 20,25-27]. Thus, CTX, RT, or adjuvant treatment is recommended even in the early stage. Harrison et al. [28] reported that the combination of RT and CTX provides the best chance of long-term survival for patients with HGNEC. However, even with comprehensive treatment, the prevalence of relapse within 2 years remains high among patients with advanced-stage disease $[25,26,29]$.

Because ovarian NETs are rare, information regarding the safety of conservative treatment designed to preserve fertility in young women is very limited. However, if the patient recognizes the risk, fertilitypreserving surgery can be considered in the early stage $[1,6]$.

Due to the rarity of the disease and the lack of systematic population-based research or registration data, treatment strategies for ovarian NETs have not been standardized. It is relatively unlikely that the tumor will be identified as an NET prior to surgery, meaning that the treatment will be based on surgical findings. 
In addition, most adjuvant treatments are based on the treatment plan for lung NETs, which consists of a combination of surgical resection and postoperative supplementation with platinum-based CTX. The most commonly used drugs include cisplatin, carboplatin, doxorubicin, epirubicin, and etoposide [25, 29]. Even in patients with relapse, the current treatment plan is still a comprehensive treatment based on platinum CTX, and postoperative adjuvant CTX is more common in patients with ovarian epithelial tumors, with relatively fewer patients receiving adjuvant RT. Surgery combined with RT and CTX plays an important role in patient prognosis, especially for those with advanced ovarian NETs. Taken together, the available evidence highlights the need to develop novel drug treatments such as molecular targeting strategies. Pembrolizumab has been used in patients with recurrent cervical and vulvar NETs and has shown promise in phase II clinical trials [30]. Therefore, clinical trials investigating the use of pembrolizumab for ovarian NETs are warranted.

We compared the characteristics of patients with different histological subtypes of ovarian NETs (LGNET vs. HGNEC). LGNET was milder, more frequently presented as stage I disease, more often involved tumors with diameters $<5 \mathrm{~cm}$, and was associated with lower rates of lymph node positivity and distant metastasis than HGNEC. Among patients with LGNET, 94.3\% were treated with surgery alone, and the 5year survival rate was as high as $93.56 \%$. These findings indicate that prognosis is relatively better among patients with ovarian LGNET than among those with HGNEC.

The present study has some limitations. Importantly, the SEER database does not include information regarding the number of chemotherapy cycles, specific chemotherapy regimens, or neoadjuvant chemotherapy regimens. In addition, the time, location, and treatments for disease recurrence are not specified, and the SEER database does not yet provide preoperative imaging data. Nonetheless, use of the SEER database was also the major strength of our study, as it provides big data regarding cases of ovarian NETs over the past 12 years. To our knowledge, our study is the largest investigation of ovarian NET conducted to date. Moreover, our data provide valuable clinical information regarding different treatment regimens and related prognostic information for patients with ovarian NETs.

In conclusion, the current research reveals that age, AJCC stage, treatment method, and histological type are independent prognostic factors for ovarian NETs. Moreover, our results indicate that prognosis is relatively good among patients with early-stage disease treated with surgery alone. However, comprehensive treatment involving surgery, CTX, and RT is required to improve prognosis in patients with advanced disease. Future studies should focus on the development of individualized treatment strategies for prolonging survival time in patients with ovarian NETs.

\section{Abbreviations}

NET: neuroendocrine tumor, ACT: atypical carcinoid tumor, SCCO: small-cell carcinoma of the ovary; LCNEC: large-cell neuroendocrine carcinoma; WHO: World Health Organization; NSCNEC: non-small-cell neuroendocrine carcinoma; LGNET: low-grade neuroendocrine tumor; HGNEC: high-grade neuroendocrine carcinoma; SEER: Surveillance, Epidemiology, and End Results; AJCC: American Joint Committee on 
Cancer; CTX: chemotherapy; RT: radiotherapy; CSS: cancer-specific survival; OS: overall survival; Cl: confidence interval; HR: hazard ratio; NSE: neuron-specific enolase; EMA: epithelial membrane antigen; MRI: magnetic resonance imaging.

\section{Declarations}

Ethics approval and consent to participate: The deidentified data in the SEER database are publicly available, and thus their use is exempt from review by the Shengjing Hospital of China Medical University Institutional Review Board.

Consent for publication: Not applicable

Availability of data and materials:The authors agree to provide our data for the reproducibility of this study in other centers if such a request is made.

Competing interests: The authors report no conflicts of interest related to this work.

Funding: No financial support was received for this study.

Authors' contributions: LP collected clinical data and wrote the paper; ZQG helped to design and revise the paper.

Acknowledgments: Not applicable

\section{References}

1. Garnder GJ, Reidy-Lagunes D, Gehrig PA. Neuroendocrine tumors of the gynecologic tract: a Society of Gynecologic Oncology (SGO) clinical document. Gynecol Oncol. 2011;122:190-8. https://doi.org/10.1016/j.ygyno.2011.04.011.

2. Guadagno EE, De Rosa G, Del B De Caro. M. Neuroendocrine tumours in rare sites: differences in nomenclature and diagnostics-a rare and ubiquitous histotype. J Clin Pathol. 2016;69:563-74. https://doi.org/10.1136/jclinpath-2015-203551.

3. Yasuoka H, Tsujimoto M, Fujita S, Kunishige I, Nishio Y, Kodama R, et al. Monoclonality of composite large cell neuroendocrine carcinoma and mucinous epithelial tumor of the ovary: a case study. Int J Gynecol Pathol 2009;28;55-8. https://dx.doi.org/10.7727\%2Fwimj.2014.010.

4. Rouzbahman M, Clarke B. Neuroendocrine tumors of the gynecologic tract: select topics. Semin Diagn Pathol. 2013;30:224-33. https://doi.org/10.1053/j.semdp.2013.06.007.

5. Eichhorn JH, Young RH. Neuroendocrine tumors of the genital tract. Am J Clin Pathol. 2001;115(Suppl.):94-112. https://doi.org/10.1309/64cw-wkgk-49ef-byd1.

6. Howitt BE, Kelly P, McCluggage WG. Pathology of neuroendocrine tumours of the female genital tract. Curr Oncol Rep. 2017;19:59. https://doi.org/10.1007/s11912-017-0617-2. 
7. National Cancer Institute Surveillance, Epidemiology and End Results Program. 2018, https://seer.cancer.gov/.Accessed 21 Sept 2020.

8. Preda VA, Chitoni M, Talbot D, Reed N, Grossman AB. Primary ovarian carcinoid: extensive clinical experience with an underrecognized uncommon entity. Int J Gynecol Cancer. 2018;28:466-71. https://doi.org/10.1097/igc.0000000000001215.

9. Burkeen G, Chauhan A, Agrawal R, Raiker R, Kolesar J, Anthony L, et al. Gynecologic large cell neuroendocrine carcinoma: a review. Rare Tumors. 2020;12:2036361320968401. https://doi.org/10.1177/2036361320968401.

10. Peng $X$, Wang $\mathrm{H}$. Primary pure large cell neuroendocrine carcinoma of the ovary: A rare case report and review of literature. Med (Baltim). 2020;99:e22474. https://doi.org/ 10.1097/MD.0000000000022474.

11. He Y, Zhao H, Li XM, Yin CH, Wu YM. Y. A clinical analysis of small-cell neuroendocrine carcinoma of the gynecologic tract: report of 20 cases. Arch Gynecol Obstet. 2019;299:543-9. https://doi.org/10.1007/s00404-018-4960-9.

12. Yang X, Chen J, Dong R. Pathological features, clinical presentations and prognostic factors of ovarian large cell neuroendocrine carcinoma: a case report and review of published literature. $J$ Ovarian Res. 2019;12:69. https://doi.org/10.1186/s13048-019-0543-z.

13. Soga J, Osaka M, Yakuwa Y. Carcinoids of the ovary: an analysis of 329 reported cases. J Exp Clin Cancer Res. 2000;19:271-80.

14. Sehouli J, Woopen H, Pavel M, Richter R, Lauterbach LK, Taube E, et al. Neuroendocrine neoplasms of the ovary: a retrospective study of the North Eastern German Society of Gynecologic Oncology (NOGGO). Anticancer Res. 2016;36:1003-9.

15. Vora M, Lacour RA, Black DR, Turbat-Herrera EA, Gu X. Neuroendocrine tumors in the ovary: histogenesis, pathologic differentiation, and clinical presentation. Arch Gynecol Obstet. 2016;293:659-65. https://doi.org/10.1007/s00404-015-3865-0.

16. Ramos P, Karnezia AN, Craig DW, Sekulic A, Russell ML, Hendricks WPD, et al. Small cell carcinoma of the ovary, hypercalcaemic type, displays frequent inactivating germline and somatic mutations in SMARCA4. Nat Genet. 2014;46:427-9. https://doi.org/10.1038/ng.2928.

17. Conlon N, Silva A, Guerra E, Jelinic P, Schlappe BA, Olvera N, et al. Loss of SMARCA4 expression is both sensitive and specific for the diagnosis of small cell carcinoma of ovary, hypercalcemic type. Am J Surg Pathol. 2016;40:395-403. https://doi.org/ 10.1097/PAS.0000000000000558.

18. Witkowski L, Goudie C, Foulkes WD, McCluggage WG. Small-cell carcinoma of the ovary of hypercalcemic type (malignant rhabdoid tumor of the ovary): a review with recent developments on pathogenesis. Surg Pathol Clin. 2016;9:215-26. https://doi.org/10.1016/j.path.2016.01.005.

19. Nasioudis D, Frey MK, Chapman-Davis E, Caputo TA, Holcomb K. Primary malignant ovarian carcinoid; management and outcomes. Gynecol Oncol. 2020;157:101-05. https://doi.org/10.1016/j.ygyno.2020.01.002. 
20. Reed NS. Neuroendocrine tumours of the gynecological tract. Curr Opin Oncol. 2016;28:412-8. https://doi.org/10.1097/CCO.0000000000000321.

21. Klimstra DS, Modlin IR, Coppola D, Lloyd RV, Suster S. The pathologic classification of neuroendocrine tumors: a review of nomenclature, grading, and staging systems. Pancreas. 2010;39:707-12. https://doi.org/10.1097/MPA.0b013e3181ec124e.

22. Oberg K, Kvols L, Caplin M, Delle Fave G, de Herder W, Rindi G, et al. Consensus report on the use of somatostatin analogs for the management of neuroendocrine tumors of the gastroenteropancreatic system. Ann Oncol. 2004;15:966-73. https://doi.org/10.1093/annonc/mdh216.

23. Vilar E, Salazar R, Pérez-García J, Cortes J, Oberg K, Tabernero J. Chemotherapy and role of the proliferation marker Ki-67 in digestive neuroendocrine tumors. Endocr Relat Cancer. 2007;14:221-32. https://doi.org/10.1677/ERC-06-0074.

24. Lou L, Zhou L, Wang W, Li H, Li Y. Atypical ovarian carcinoid tumor with widespread skeletal metastases: a case report of multiple endocrine neoplasia type 1 in a young woman. BMC Cancer. 2019;19:1107. https://doi.org/10.1186/s12885-019-6332-7.

25. Nasioudis D, Chapman-Davis E, Frey MK, Caputo TA, Witkin SS, Holcomb K. Small cell carcinoma of the ovary: a rare tumor with a poor prognosis. Int J Gynecol Cancer. 2018;28:932-8. http://dx.doi.org/10.1097/IGC.0000000000001243.

26. Safini F, Jouhadi H, El Attar H. Primary large cell neuroendocrine carcinoma of the ovary: A rare entity. Gulf J Oncol. 2021;1:82-5.

27. Atienza-Amores M, Guerini-Rocco E, Soslow RA, Park KJ, Weigelt B. Small cell carcinoma of the gynecologic tract: a multifaceted spectrum of lesions. Gynecol Oncol. 2014;134:410-8. https://doi.org/10.1016/j.ygyno.2014.05.017.

28. Harrison ML, Hoskins P, du Bois A, Quinn M, Rustin GJS, Ledermann JA, et al. Small cell of the ovary, hypercalcemic type: analysis of combined experience and recommendation for management. A GCIG study. Gynecol Oncol. 2006;100:233-8. https://doi.org/10.1016/j.ygyno.2005.10.024.

29. Reed NS, Pautier P, Åvall-Lundqvist E, Choi CH, du Bois A, Friedlander M, et al. Gynecologic Cancer InterGroup (GCIG) consensus review for ovarian small cell cancers. Int J Gynecol Cancer. 2014;24:30-4. http://dx.doi.org/10.1097/IGC.0000000000000293.

30. Frumovitz M, Westin SN, Salvo G, Zarifa A, Xu M, Yap TA, et al. Phase Il study of pembrolizumab efficacy and safety in women with recurrent small cell neuroendocrine carcinoma of the lower genital tract. Gynecol Oncol. 2020;158:570-5. https://doi.org/10.1016/j.ygyno.2020.05.682.

\section{Tables}

Due to technical limitations, table 1 to 4 is only available as a download in the Supplemental Files section.

\section{Figures}


A

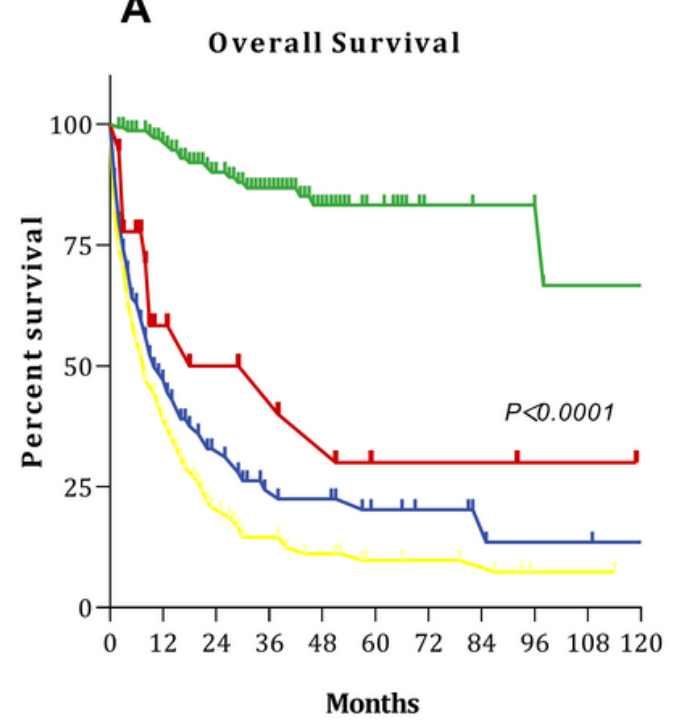

$\perp$ Stagel

$\perp$ Stagell

$\perp$ StagellI StagelV

\section{Figure 1}

Survival curves at each stage: (A) overall survival (OS); (B) cancer-specific survival (CSS).

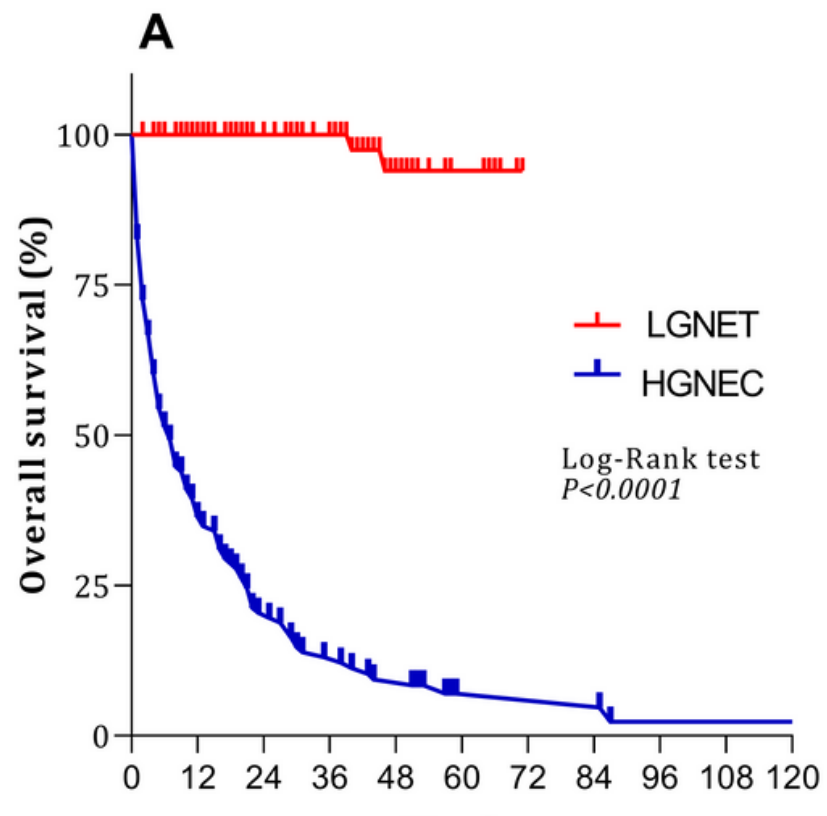

Months
B
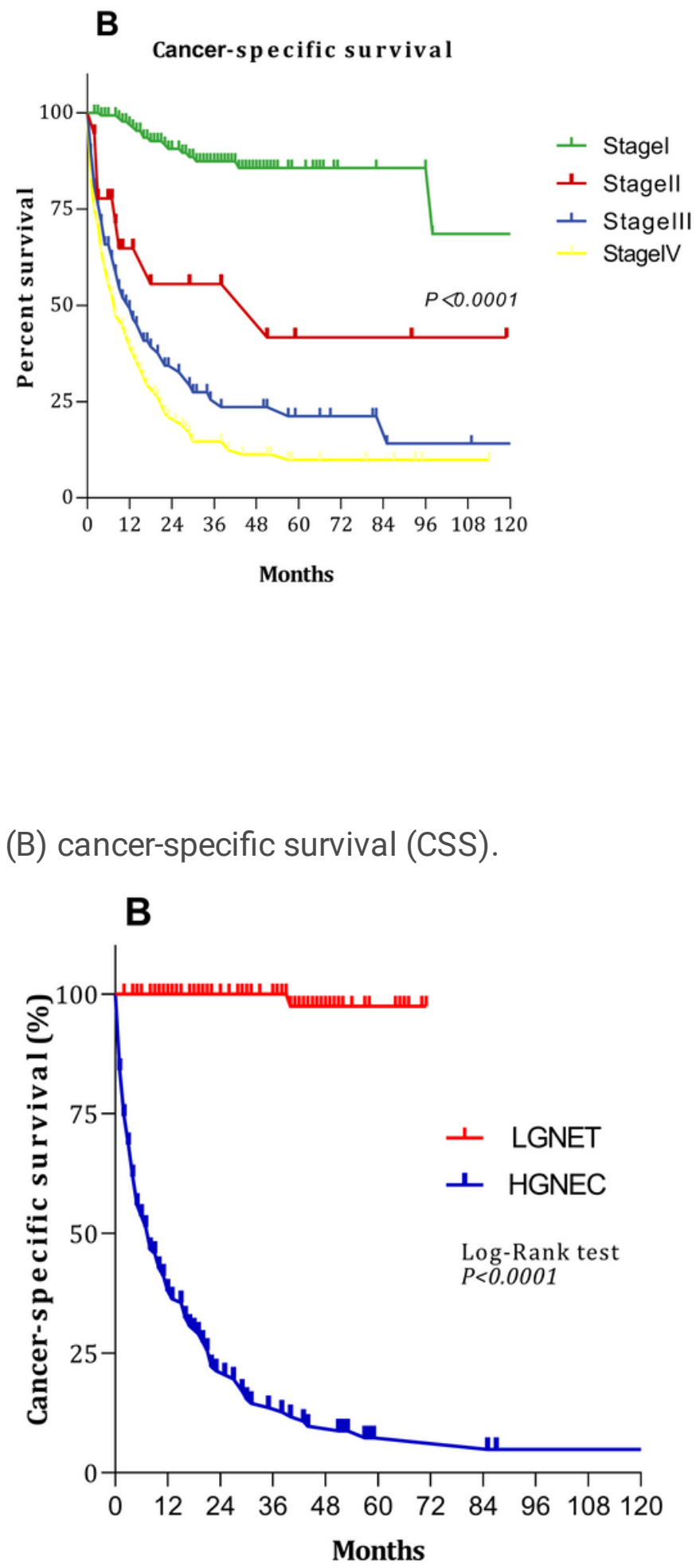

Figure 2

Survival curves for patients with low-grade neuroendocrine tumors (LGNET) and high-grade neuroendocrine carcinoma (HGNEC): (A) overall survival (OS); (B) cancer-specific survival (CSS). 

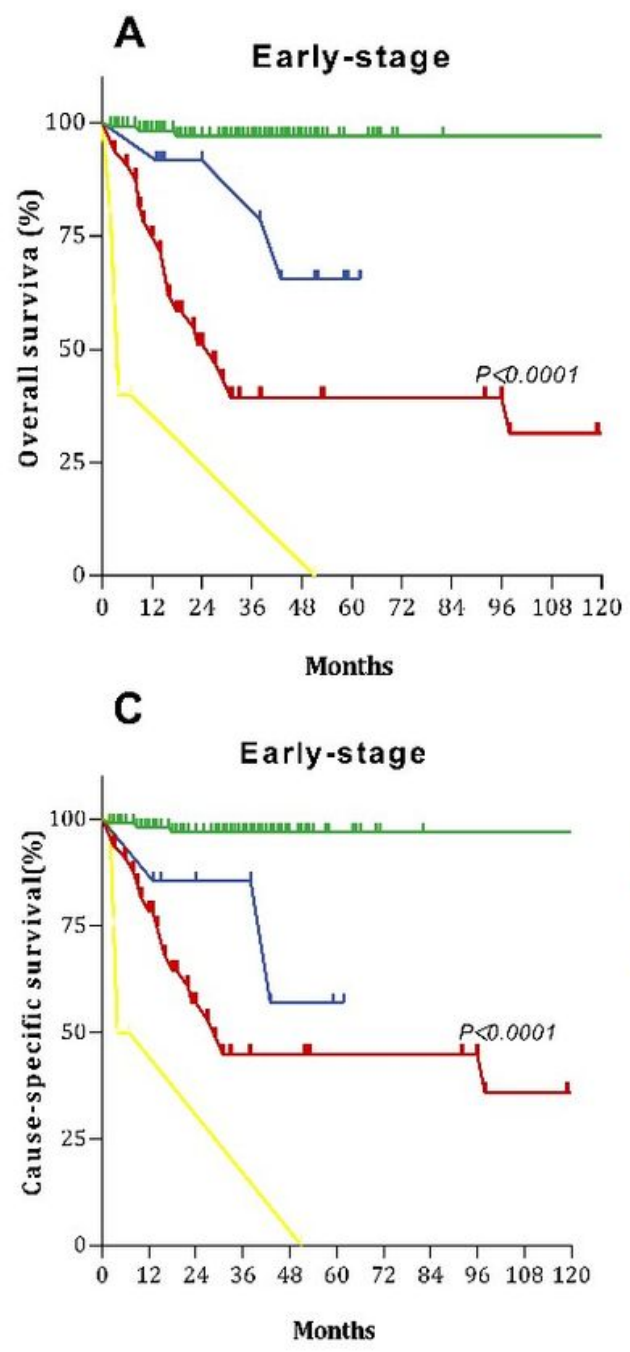

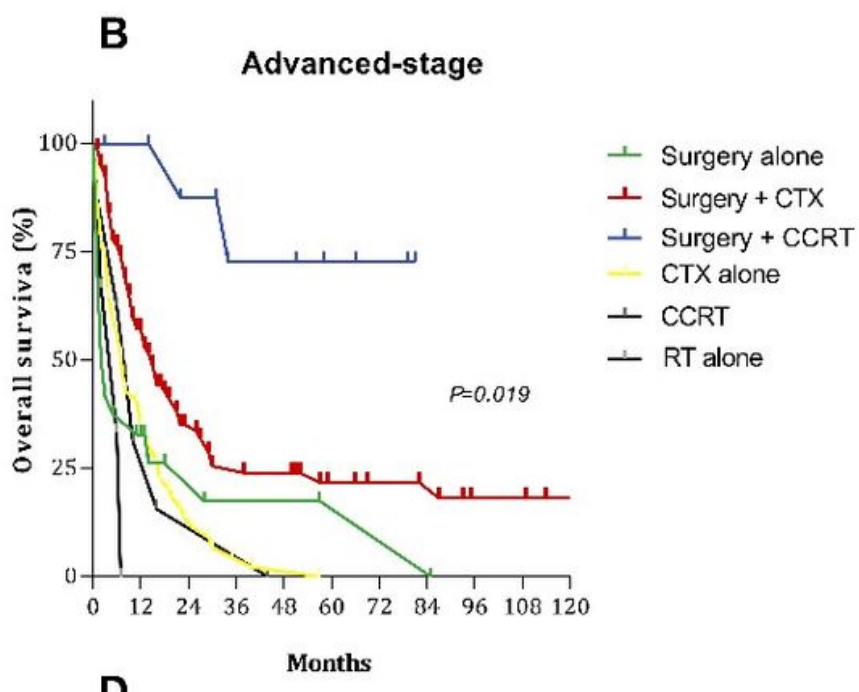

+ Surgery alone

土 Surgery + CTX

$\perp$ Surgery + CCRT

CTX alone $\perp$ Surgery + CTX

$\perp$ Surgery + CCRT

CTX alone
D

Advanced-stage

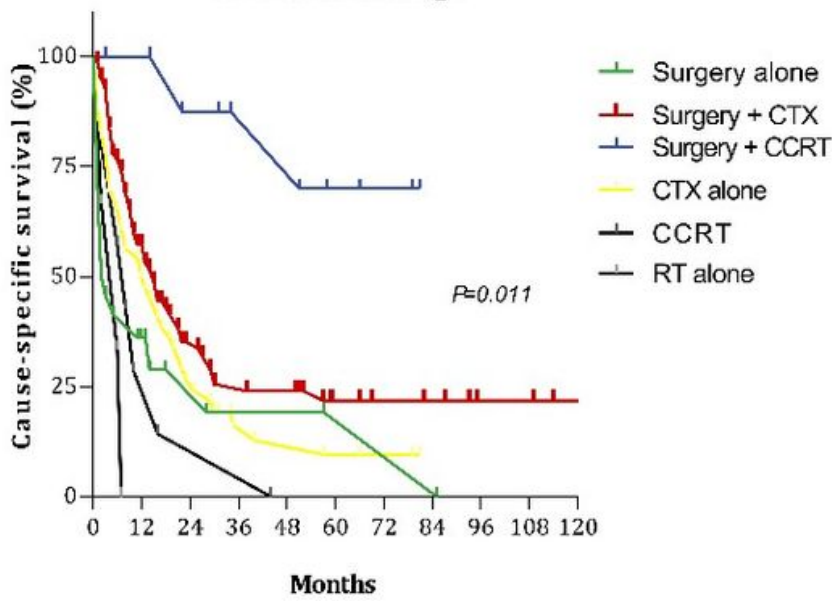

\section{Figure 3}

Survival curves for patients with early- and advanced-stage disease for different treatment regimens: (A) overall survival (OS) in the early stage; (B) overall survival (OS) in the advanced stage; (C) cancer-specific survival (CSS) in the early stage; (D) cancer-specific survival (CSS) in the advanced stage.

\section{Supplementary Files}

This is a list of supplementary files associated with this preprint. Click to download.

- Table1.xlsx

- table2.xIsx

- Table3.xlsx

- table4.xlsx

- supplementalfigure1.tif

- supplementaltable1.xlsx 